\title{
EDITORIAL
}

\section{Focus on ultrasound in intensive care}

\author{
Giovanni Volpicelli ${ }^{1^{*}}$, Paul Mayo ${ }^{2}$ and Serena Rovida ${ }^{3}$
}

C 2020 Springer-Verlag GmbH Germany, part of Springer Nature

Over the past two decades, the amount of literature on point of care ultrasound (PoCUS) has been increasing rapidly. Nowadays, a growing body of evidence strongly supports its role in several fields with particular interest to the acute care settings. This article will review some recent literature that may be of interest to the critical care community. Our literature review mainly focuses on thoracic PoCUS, including eight articles on lung ultrasound (LUS), six articles on echocardiography and one article on diaphragm ultrasound. We also cite an important review on brain ultrasound, one article on multiorgan PoCUS and two articles on procedure guidance.

\section{Lung ultrasound}

LUS is a key tool for intensivists due to its ease of use and utility in critically ill patients. A narrative review outlined the main applications of LUS for a wide variety of lung conditions as a valuable alternative to chest computerized tomography (CT) scan and chest X-ray (CXR) [1]. The authors of this article identified some advantages of LUS that include the superiority of LUS when compared to CXR to detect pneumothorax, pleural effusion, pneumonia and interstitial syndromes and to establish diagnosis of acute dyspnea. In addition, LUS had utility for recognition of different $B$ line patterns to allow differentiation of acute cardiogenic pulmonary edema from acute respiratory distress syndrome (ARDS). Moreover, LUS allowed measurement of a score that correlates with increase in lung density and loss of lung aeration, which may be relevant to management of patients with ARDS. Finally, LUS in combination with other aspects of ultrasonography may be useful in the diagnosis of pulmonary embolism.

\footnotetext{
*Correspondence: giovi.volpicelli@gmail.com

${ }^{1}$ Department of Emergency Medicine, San Luigi Gonzaga University Hospital, Turin, Italy
}

Full author information is available at the end of the article
The diagnostic accuracy of LUS in identifying type, extension and distribution of lung lesions was compared to chest CT in a group of adult patients with ARDS [2]. Two anterior, two lateral and two posterior lateral areas on each hemithorax were scored. LUS was highly accurate for both pattern recognition and distribution of the disease pattern in all regions when compared to chest CT with accuracy being highest in the lateral and posterior lateral lung regions. The authors cautioned that a limitation of LUS is that it only detects lung conditions that are adjacent to the pleural surface.

Ferrè et al. investigated the utility of LUS to detect weaning-induced pulmonary oedema (WIPO) during 62 spontaneous breathing trials in 42 patients [3]. WIPO occurred in 17 cases, and all these patients failed extubation. The best pattern of LUS predicting WIPO and failure of extubation was an increase in at least six B lines in the four anterior chest areas.

Although it is well established that LUS is far more sensitive than anterior-posterior CXR for the diagnosis of pneumothorax, the visual identification of lung sliding is qualitative and may be challenging for the inexperienced operator in the acute setting. Duclos et al. reported an alternative method to identify lung sliding based on longitudinal pleural strain value and speckle tracking analysis [4]. In this retrospective study, the respirophasic longitudinal pleural strain pattern on the side of the pneumothorax was compared to the contralateral normal side. Pleural strain measurement was able to discriminate lung sliding from no lung sliding with excellent performance characteristics. Speckle tracking permitted a quantitative assessment of lung respiratory movement.

Volpicelli pointed out peculiar features of LUS for the diagnosis of pneumothorax [5]. The lung point is the sonographic demonstration of the transition between the pneumothorax and the lung. It is visualized when a respiratory pattern (sliding) intermittently replaces the motionless pleura. This specific sign cannot be mimicked. In cases of regional altered ventilation, a motionless

\section{Springer}


pleura is also visualized but without interposition of the respiratory sliding.

\section{Echocardiography}

A comprehensive review by Vieillard-Baron et al. supported the utility of critical care echocardiography for the management of hemodynamic failure [6]. Cholley advocated the importance of including aortic velocity-time integral analysis as a standard measurement of hemodynamic function [7].

A retrospective study on 56 patients in the ICU compared the accuracy of critical care transesophageal echocardiography (TEE) performed by intensivists to TEE or transthoracic echocardiography (TTE) performed by cardiologists [8]. The results of the study indicate that TEE performed by intensivists yields the similar results to TEE by cardiologists. These two articles indicate that intensivist-performed TTE and TEE should be a standard component of critical care practice, as they are feasible and accurate modalities for answering diagnostic and hemodynamic questions in critically ill patients.

A randomized controlled clinical trial evaluated the use of continuous hemodynamic monitoring with TEE (hTEE) versus usual care in 550 critically ill patients with shock [9]. The hTEE monitoring in patients with circulatory shock did not shorten the time to resolution of signs of hypoperfusion, duration of organ support, length of stay and mortality at 6 days. However, when examining the first $72 \mathrm{~h}$, the group with hTEE monitoring showed a statistically significant reduction in hemodynamic instability. In a single-center retrospective analysis of 25 adult patients with cardiac arrest, Fair et al. explored the benefit of TEE during resuscitation [10]. TEE was efficient and provided the shortest mean compression pauses duration (up to 9 s) compared with TTE (up to 19 s). An original study of Geri et al. showed that TEE monitoring in ICU patients with septic shock can identify cardiovascular clusters that are potentially useful to orientate fluid infusion, vasopressors and inotropic treatment and predict prognosis [11]. The combination of echocardiographic and clinical parameters allows the differentiation of five phenotypes, based on the assessment of left ventricle (LV) or right ventricle depression, LV hyperkinesia, fluid responsiveness and persistent hypovolemia.

\section{Diaphragm ultrasound}

Diaphragm ultrasound is emerging as a new useful technique to assess and monitor the dynamic of respiratory function in critically ill patients. A new study showed that a PoCUS evaluation of diaphragm thickening fraction in preoperative cardiac surgery may predict postoperative pulmonary complications [12]. Patients developing postoperative pneumonia, prolonged invasive ventilation, persistent atelectasis were those with significantly lower thickening fraction.

\section{Mutiorgan ultrasound}

A multiorgan PoCUS assessment finds its relevance in critically ill pregnant and postpartum patients. In this regard, Blanco et al. proposed an algorithm 'head to toe' to diagnose the relevant neurological, pulmonary, cardiac and abdominal emergencies in different gestational periods [13].

\section{Brain ultrasound}

PoCUS enables early diagnosis of neurological conditions by detecting indirect signs of intracranial hypertension such as brain midline shift and increase in the optic nerve sheath diameter. It also allows monitoring of changes in the vascular flow autoregulation during systemic diseases like sepsis, acute hepatic failure and brain trauma and can be used in neuro- and general ICUs, the trauma unit, the emergency department and the operating room [14].

\section{Procedural guidance}

In addition to diagnostic imaging, PoCUS can safely guide invasive procedures. A comprehensive review addresses the state of the art and the evidences on the ultrasound-guided vascular access in the ICU [15]. There is a clear advantage for PoCUS guidance versus the landmark technique at the site of internal jugular vein (IJV), demonstrated by several studies. Other ultrasound-guided approaches, including the subclavian vein (SCV), axillary vein and femoral vein, are also increasingly supported by evidence. Shin et al. demonstrated a very low complication rates for both IJV and SCV ultrasound-guided catheterization [16]. This new study demonstrated that catheterization of both veins is safe when guided by ultrasound, without superiority of the subclavian approach. A standard approach should anyway follow a pragmatic protocol comprising of sequential steps to explore the site, guide the procedure and confirm the correct placement [15].

\section{Scientific evidence on PoCUS}

Despite a growing evidence that PoCUS could replace CXR, translation of scientific evidence into clinical practice is still considered by some commentators to be too low to support the adoption of PoCUS as a standard clinical tool. Two editorials expressed concern that there was insufficient scientific evidence on the efficacy of PoCUS and that there was need for large randomized controlled trials (RCT) before PoCUS could be incorporated into clinical practice $[17,18]$. In answer to this concern, Pivetta et al. published a multicenter RCT on 518 dyspneic patients that demonstrated the superior diagnostic 
accuracy of LUS compared to CXR when these two tests were integrated into the diagnostic workup for acute decompensated heart failure [19]. Although the study did not investigate differences in patient outcome that would have required the use of a complex RCT protocol, these data further contribute to the existing evidence of the diagnostic efficacy of using LUS in the acute care setting.

In conclusion, several applications of PoCUS in the ICU are of interest to the critical care community. The literature is progressing rapidly yearly. The main topics of interest are the thoracic applications, including lung, cardiac and diaphragm ultrasound, followed by brain ultrasound and procedural guidance. The trend of the new studies is the demonstration of the diagnostic accuracy of new PoCUS methods and their impact in the daily practice of critical care.

\section{Author details \\ ${ }^{1}$ Department of Emergency Medicine, San Luigi Gonzaga University Hos- pital, Turin, Italy. ${ }^{2}$ Division of Pulmonary, Critical Care and Sleep Medicine, Northwell Health, Zucker School of Medicine at Hofstra/Northwell, New Hyde Park, Hempstead, NY, USA. ${ }^{3}$ Department of Emergency Medicine, Linköping University Hospital, Linköping, Sweden.}

\section{Compliance with ethical standards}

\section{Conflicts of interest}

The authors declare they have no conflicts of interest.

\section{Publisher's Note}

Springer Nature remains neutral with regard to jurisdictional claims in published maps and institutional affiliations.

Received: 20 February 2020 Accepted: 24 March 2020

Published online: 31 March 2020

\section{References}

1. Mayo PH, Copetti R, Feller-Kopman D, Mathis G, Maury E, Mongodi S, Mojoli F, Volpicelli G, Zanobetti M (2019) Thoracic ultrasonography: a narrative review. Intensive Care Med 45:1200-1211

2. Chiumello D, Umbrello M, Sferrazza Papa GF, Angileri A, Gurgitano M, Formenti P, Coppola S, Froio S, Cammaroto A, Carrafiello G (2019) Global and regional diagnostic accuracy of lung ultrasound compared to CT in patients with acute respiratory distress syndrome. Crit Care Med 47:1599-1606

3. Ferré A, Guillot M, Lichtenstein D, Mezière G, Richard C, Teboul JL, Monnet X (2019) Lung ultrasound allows the diagnosis of weaning-induced pulmonary oedema. Intensive Care Med 45:601-608

4. Duclos G, Bobbia X, Markarian T, Muller L, Cheyssac C, Castillon S, Resseguier N, Boussuges A, Volpicelli G, Leone M, Zieleskiewicz L (2019)
Speckle tracking quantification of lung sliding for the diagnosis of pneumothorax: a multicentric observational study. Intensive Care Med $45: 1212-1218$

5. Volpicelli G (2019) A lung point that is not a lung point. Intensive Care Med 45:1326

6. Vieillard-Baron A, Millington SJ, Sanfilippo F, Chew M, Diaz-Gomez J, McLean A, Pinsky MR, Pulido J, Mayo P, Fletcher N (2019) A decade of progress in critical care echocardiography: a narrative review. Intensive Care Med 45:770-788

7. Cholley B (2019) Echocardiography in the intensive care unit: beyond "eyeballing". A plea for the broader use of the aortic velocity-time integral measurement. Intensive Care Med 45:898

8. Lau V, Priestap F, Landry Y, Ball I, Arntfield R (2019) Diagnostic accuracy of critical care transesophageal echocardiography vs cardiology-led echocardiography in ICU patients. Chest 155:491-501

9. Merz TM, Cioccari L, Frey PM, Bloch A, Berger D, Zante B, Jakob SM, Takala J (2019) Continual hemodynamic monitoring with a singleuse transesophageal echocardiography probe in critically ill patients with shock: a randomized controlled clinical trial. Intensive Care Med 45:1093-1102

10. Fair J 3rd, Mallin MP, Adler A, Ockerse P, Steenblik J, Tonna J, Youngquist ST (2019) Transesophageal echocardiography during cardiopulmonary resuscitation is associated with shorter compression pauses compared with transthoracic echocardiography. Ann Emerg Med 73:610-616

11. Geri G, Vignon P, Aubry A, Fedou AL, Charron C, Silva S, Repessé X, Vieillard-Baron A (2019) Cardiovascular clusters in septic shock combining clinical and echocardiographic parameters: a post hoc analysis. Intensive Care Med 45:657-667

12. Cavayas YA, Eljaiek R, Rodrigue É, Lamarche Y, Girard M, Wang HT, Levesque S, Denault AY (2019) Preoperative diaphragm function is associated with postoperative pulmonary complications after cardiac surgery. Crit Care Med 47:e966-e974

13. Blanco P, Abdo-Cuza A (2019) Point-of-care ultrasound in the critically ill pregnant or postpartum patient: what every intensivist should know. Intensive Care Med 45:1123-1126

14. Robba C, Goffi A, Geeraerts T, Cardim D, Via G, Czosnyka M, Park S, Sarwal A, Padayachy L, Rasulo F, Citerio G (2019) Brain ultrasonography: methodology, basic and advanced principles and clinical applications. A narrative review. Intensive Care Med 45:913-927

15. Schmidt GA, Blaivas M, Conrad SA, Corradi F, Koenig S, Lamperti M, Saugel B, Schummer V, Slama M (2019) Ultrasound-guided vascular access in critical illness. Intensive Care Med 45:434-446

16. Shin HJ, Na HS, Koh WU, Ro YJ, Lee JM, Choi YJ, Park S, Kim JH (2019) Complications in internal jugular vs subclavian ultrasound-guided central venous catheterization: a comparative randomized trial. Intensive Care Med 45:968-976

17. Bistola V, Polyzogopoulou E, Ikonomidis I, Parissis J (2019) Lung ultrasound for the diagnosis of acute heart failure: time to upgrade current indication? Eur J Heart Fail 21:767-769

18. Corcoran JP, Laursen CB (2019) COUNTERPOINT: should point-of-care ultrasound examination be routine practice in the evaluation of the acutely breathless patient? No. Chest 156:426-428

19. Pivetta E, Goffi A, Nazerian P, Castagno D, Tozzetti C, Tizzani P, Tizzani M, Porrino G, Ferreri E, Busso V, Morello F, Paglieri C, Masoero M, Cassine E, Bovaro F, Grifoni S, Maule MM, Lupia E (2019) Lung ultrasound integrated with clinical assessment for the diagnosis of acute decompensated heart failure in the emergency department: a randomized controlled trial. Eur J Heart Fail 21:754-766 